Article

\title{
Synthesis of Negatively Charged Polyol-Functional PSF Membranes with Good Hydrophilic and Efficient Boron Removal Properties
}

\author{
Jinbo Jin ${ }^{1,2}$, Xilan $\mathrm{Du}^{2}$, Jie $\mathrm{Yu}^{1,2, * \mathbb{C}}$, Shuhao Qin ${ }^{1,2, *}$, Min $\mathrm{He}^{1}{ }^{1}$, Kaizhou Zhang ${ }^{2}$ and \\ Jingkui Yang ${ }^{2}$ \\ 1 College of Materials and Metallurgy, Guizhou University, Guiyang 550025, China; jinjinbo66@163.com (J.J.); \\ hemin851@163.com (M.H.) \\ 2 National Engineering Research Center for Compounding and Modification of Polymeric Materials, \\ Guiyang 550014, China; mydx1123@163.com (X.D.); Try-boat@163.com (K.Z.); jkyang2006@163.com (J.Y.) \\ * Correspondence: yujiegz@126.com (J.Y.); qinshgz@163.com (S.Q.)
}

Received: 1 April 2019; Accepted: 25 April 2019; Published: 1 May 2019

check for updates

\begin{abstract}
Boron removal remains a major barrier to water purification, it is important to develop a specialized adsorption membrane for boron removal. By means of a simple and effective method, a hydrophilic membrane for boron removal with a polyhydroxy functional group on the surface was prepared. Firstly, a polysulfone (PSF) membrane was modified by co-depositing polyethyleneimine (PEI) with dopamine (DA) in one-step to produce amine-rich surfaces, then the DA/PEI-functionalized membranes were reacted with glycidol, with the prepared membranes corresponding to PSF-PDA/PEI membranes and PSF-diol membranes. The prepared membranes were characterized by water-uptake, FTIR, (X-ray photoelectron spectroscopy) XPS, (Field emission scanning electron microscope) FESEM, and zeta potential measurements. The hydrophilicity of the membrane was characterized by the static water contact angle (WCA) test. In addition, we systematically studied the impact of initial boron concentration, chelating time, and $\mathrm{pH}$ value on boron removal performance. The results showed that the PSF-diol membrane had strong hydrophilicity with a WCA of about $38^{\circ}$. The maximum adsorption capacity of boron appeared to be $1.61 \mathrm{mmol} / \mathrm{g}$ within $10 \mathrm{~min}$ at a boron concentration of $300 \mathrm{mg} / \mathrm{L}$. Adsorption kinetics showed that saturation adsorption can be achieved in minutes at the initial concentration of $5 \mathrm{mg} / \mathrm{L}$, which is beneficial to a rapid filtration process.
\end{abstract}

Keywords: affinity surface; polyhydroxy; boron adsorption; superhydrophilic

\section{Introduction}

Boron is one of the essential trace elements for plants and animals [1]. Appropriate boron can promote the transport of plant carbohydrates, regulate the synthesis of sugars, and promote cell division [2,3]. However, the difference between boron deficiency and boron poisoning is very small. When the concentration of boron in soil is above $2 \mathrm{mg} / \mathrm{L}$, the plant will show boron poisoning, while when the boron content is lower than $1 \mathrm{mg} / \mathrm{L}$, the plant will show boron deficiency $[4,5]$. For humans, excessive intake of boron can seriously affect people's health, causing symptoms such as nausea, vomiting, headache, diarrhea, kidney damage, and even reproductive nervous system diseases [6]. Therefore, researchers have studied the problem of boron removal and proposed a variety of boron removal methods. So far, there are mainly adsorption method, precipitation method, liquid-liquid extraction method, electro-dialysis, ion-exchange resin method, electro-coagulation method, and membrane separation methods [7-10]. However, because of the low boron content, small radius, low electro-negativity, and other special properties, boron removal technology remains a technical problem [11,12]. 
Membrane separation technology is a new and efficient separation technology in water treatment, but it suffers from limitations such as the size of the separated substance and charge discrimination [13]. Affinity membrane separation is a new method with high selectivity and efficiency for the removal and enrichment of trace substances $[14,15]$. Therefore, combining the advantages of membrane technology and the characteristics of the boron element, it is necessary to develop a specialized adsorption membrane for boron removal. Polyhydroxy structure has been proved to have effective complexation with boron [16,17]. Shi et al. [15] blended a glycopolymer and polysulfone (PSF) for boron adsorption, and the results showed that boron uptake reached $0.2 \mathrm{mmol} / \mathrm{g}$. By comparing the ligand structures of polyhydroxy polymers, Du et al. [18] found that the presence of the amino group and the branched chain structure are beneficial to improve the complexing efficiency. Recently, hyperbranched-polyol-tethered membranes were proved to be highly effective and fast adsorbent, but their preparation conditions are more demanding $[19,20]$.

Dopamine (DA) is widely used to modify membrane materials due to its excellent auto-agglutination and hydrophilic properties [21,22]. The amino and carbonyl groups of polydopamine (PDA) can induce secondary modification reactions, which have attracted extensive attention [23,24]. The amino group of hyperbranched polyethyleneimine (PEI) can react with DA with Schiff base and Michael addition, so DA/PEI was deposited on the surface of a (Polyvinylidenefluoride) PVDF membrane surface; the layer can improve hydrophilicity and oil-water separation efficiency [25]. It was found that the PDA/PEI coating possesses a superhydrophilic membrane surface and the oil rejection is more than $98 \%$ [26].

Combined with the above experiments, we introduce a simple and highly efficient membrane for boron removal. Firstly, PSF membranes were modified by DA and PEI in one-step to produce amine-rich surfaces, then the DA/PEI-functionalized membranes were reacted with glycidol, so a composite membrane with polyhydroxy structure was obtained, with the prepared membranes corresponding to PSF-PDA/PEI membranes and PSF-diol membranes. The scheme is shown in Figure 1. The prepared membranes were confirmed by water-uptake measurements, FTIR, XPS, FESEM, and zeta potential tests. The hydrophilicity of the membrane was characterized by the static water contact angle (WCA) test. In addition, we systematically studied the impact of initial boron concentration, chelating time, and $\mathrm{pH}$ value on boron removal performance. We also used Freundlich and Langmuir isothermal adsorption models to fit the boron adsorption properties of thermodynamics.

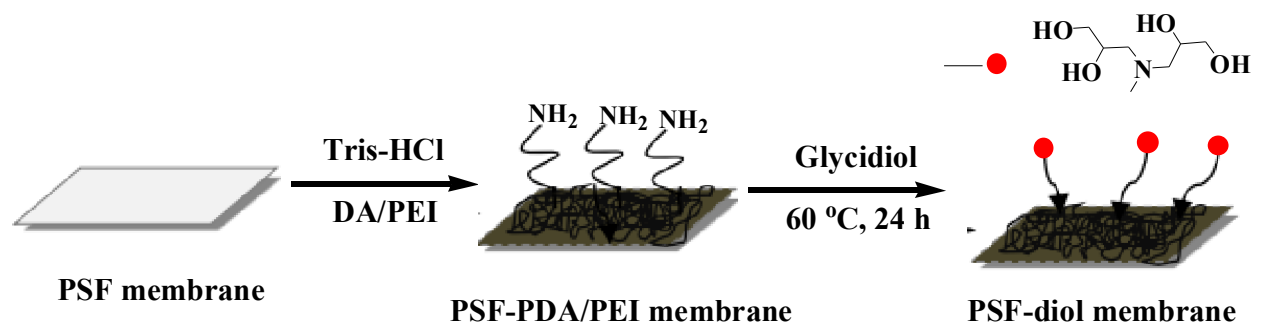

Figure 1. Schematic illustration for preparing polysulfone (PSF) composite membranes. DA—dopamine; PDA-polydopamine; PEI-polyethyleneimine.

\section{Experiments}

\subsection{Materials}

Polysulfone (PSF, $\mathrm{M}_{\mathrm{n}}=58,000 \mathrm{~g} / \mathrm{mol}$, BASF, Shanghai, China) and PEI $\left(\mathrm{M}_{\mathrm{w}}=6500 \mathrm{~g} / \mathrm{L}\right)$ were supplied by Sigma-Aldrich (Shanghai, China). Glycidol (97\%), dopamine hydrochloride (DA-HCl) $(98 \%)$, and tris(hydroxymethyl) aminomethane (Tris) were obtained from J\&K (Beijing, China). Polyvinylpyrrolidone (PVP) and N-methylpyrrolidone (NMP) were purchased from Sinopharm Group Chemical Reagents Co., Ltd. (Beijing, China). All other chemicals were analytical grade and were used without further purification. 


\subsection{Procedure of PSF-PDA/PEI Membrane Preparation}

In this paper, a PSF membrane was prepared following the steps in reference [18] with a mass ratio of PSF/PVP/NMP $=18 / 75 / 7$. The PSF-PDA/PEI membrane was prepared in the following steps. Firstly, the PSF membrane was washed clean and immersed in Tris buffer solutions $(15 \mathrm{mM}, \mathrm{pH}=8)$. Then DA and PEI were added into the buffer solutions with different mass ratios. The reaction was carried out at $25^{\circ} \mathrm{C}$ for $10 \mathrm{~h}$. After reaction, the prepared membrane was cleaned with deionized water to remove adsorbed molecules.

\subsection{Procedure of PSF-Diol Membrane Preparation}

The PSF-diol membrane was prepared by the reaction of amino groups on the PSF-PDA/PEI membrane with glycidol. At first, the PSF-PDA/PEI membrane was immersed in $100 \mathrm{~mL}$ of ethanol solution in $500 \mathrm{~mL}$ three-necked flasks, then glycidol was added dropwise to the solution at a $60^{\circ} \mathrm{C}$ reflux reaction for $24 \mathrm{~h}$ with magnetic stirring [27]. After that, the membrane was washed thoroughly with ethanol and ultrapure water before testing.

\subsection{Membrane Characterization}

The surface chemical compositions of the original and modified PSF membranes were analyzed by XPS (Thermo Fisher, K-alpha, Shanghai, China) and FTIR (Bruker, Vector-22, Beijing, China) measurements. The surface and cross morphology of the membrane was characterized by FESEM (Quanta FEG250, Beijing, China) tests. A zeta potential analyzer was used to characterize the surface charge characteristics of the membranes (Shanghai, China). The static water contact angles (WCA) of PSF, PSF-PDA/PEI, and PSF-diol membranes were calculated by a Kruss instrument (Hamburg, Germany).

\subsection{Water-Uptake Test}

The water-uptake test was used to character the water absorption mass of the PSF-PDA/PEI membrane after immersion in deionized water for $24 \mathrm{~h}$. The water uptake can be calculated by the equation:

$$
W_{\text {up }}=\frac{M_{\text {wet }}-M_{\text {dry }}}{M_{\text {dry }}} \times 100 \% \text {, }
$$

where $W_{\text {up }}$ stands for the water uptake (\%), $M_{\text {dry }}$ and $M_{\text {wet }}$ represent the mass of the membrane before and after water absorption, respectively.

\subsection{The Water Flux Test}

The water fluxes of the original membrane and the modified membranes were measured with a cross-flow device at $0.1 \mathrm{MPa}, 25^{\circ} \mathrm{C}$. The membrane was cleaned and pressurized before testing. The water flux was measured by the following equation:

$$
F=\frac{V}{S \cdot t^{\prime}}
$$

where $F$ represents the water flux $\left(\mathrm{L} / \mathrm{m}^{2} \mathrm{~h}\right), V$ is the volume $(\mathrm{L})$ of water through the device in a given period of time (h), $S$ is the effective area $\left(\mathrm{cm}^{2}\right)$ of the membrane, and $t$ is the penetration time $(\mathrm{h})$.

\subsection{Boron Adsorption Experiments}

A certain mass of PSF-diol membrane was cleaned with methanol, and then the membrane was put into a solution with a known boron concentration, followed by a shock adsorption reaction for $3 \mathrm{~h}$. Finally, the boron content was tested by the curcumin method [18]. The quantitative value of boron adsorbed is calculated using the following equation: 


$$
q=\frac{\left(C_{0}-C_{\mathrm{eq}}\right) V}{m M}
$$

where $C_{0}$ is the initial boron concentration $(\mathrm{mg} / \mathrm{L})$, and $C_{\text {eq }}$ represents the boron concentration at the adsorption equilibrium $(\mathrm{mg} / \mathrm{L}) . V$ could be the volume of the solution $(\mathrm{L})$ and $m$ is the mass of the membrane in the dry state $(\mathrm{g}) . M$ is the molecular weight of the boric acid $(\mathrm{g} / \mathrm{mol})$.

\section{Results and Discussion}

\subsection{Water Uptake of Membranes}

We can see from Figure 2 that when the concentration of DA and PEI is $1 \mathrm{~g} / \mathrm{L}$, the water uptake reaches the optimal level. When the concentration increased, the water absorption rate changes little. High water uptake represents high hydrophilic, therefore, when the concentration of DA/PEI is 1:1 g/L, the prepared membrane has good hydrophilicity properties.

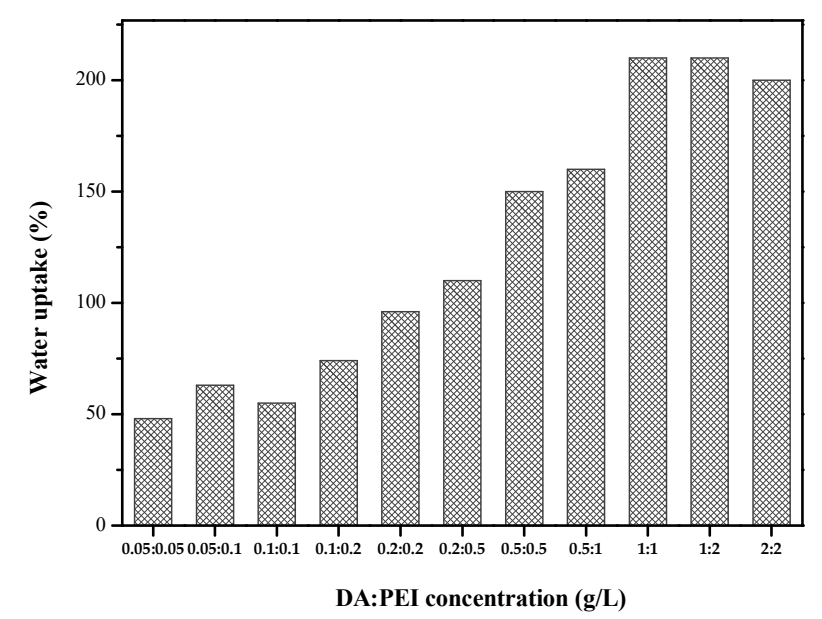

Figure 2. Water uptake of the DA/PEI-modified PSF membranes.

Under the condition of DA/PEI concentration of $1: 1 \mathrm{~g} / \mathrm{L}$, we went on to investigate the influence of deposition time on the water-uptake performance of the PSF-PDA/PEI membranes. As can be seen from Figure 3, when the deposition time was $10 \mathrm{~h}$, the water uptake of composite membranes reached saturation. With the extension of deposition time, the water uptake did not increase significantly. To get the optimum test condition, we selected the deposition time as $10 \mathrm{~h}$ and the concentration ratio of $\mathrm{DA} / \mathrm{PEI}$ is $1: 1 \mathrm{~g} / \mathrm{L}$ as a second reaction.

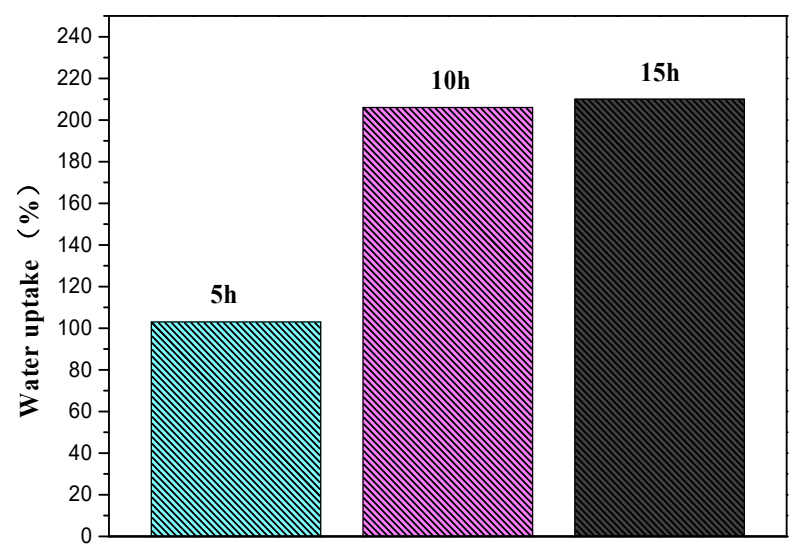

Figure 3. Water uptake of PSF-PDA/PEI with selected DA/PEI concentration (1:1 g/L) membranes at different deposition times. 


\subsection{Membrane Surface Structure Characterization}

\subsubsection{Surface Chemical Composition}

The FTIR spectra of PSF, PSF-PDA/PEI, and PSF-diol membranes are shown in Figure 4. Compared with the original film, the vibration peak of the $C=C$ double bond on the benzene ring at $1650 \mathrm{~cm}^{-1}$ of the composite membrane was enhanced, which was caused by the introduction of dopamine and polyethyleneimine on the surface of the composite membrane. In addition, the stretching vibration peaks of $\mathrm{O}-\mathrm{H}$ and $\mathrm{N}-\mathrm{H}$ appeared at $3300 \mathrm{~cm}^{-1}$ of the composite membrane, which also proved the successful preparation of the composite membrane [27,28]. Due to the residual PVP in the original PSF membrane, a weak carbonyl absorption peak appears. However, the peak intensity of $\mathrm{C}=\mathrm{O}$ was significantly increased after the co-deposition of DA/PEI. All these phenomena indicate that dopamine and polyethyleneimine successfully deposited on the surface of the polysulfone membrane.

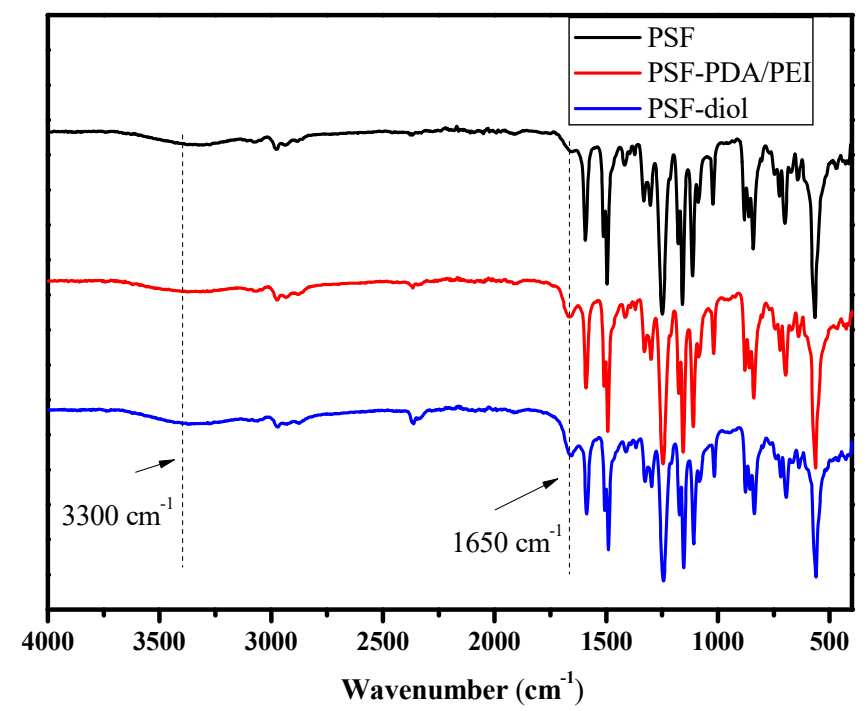

Figure 4. ATR-FTIR spectra of the PSF, PSF-PDA/PEI, and PSF-diol membranes.

We further characterized the surface element content of the base and composite membranes by XPS analysis. Figure 5 shows the XPS spectra of the original and composite membranes. As shown in Figure 5, a C1s emission peak appeared in the primary membrane at $285.1 \mathrm{eV}$. Due to the presence of the sulfone group in the primary membrane, strong emission peaks belonging to S2p and O1s appeared at 168.6 and $532.1 \mathrm{eV}$, respectively. Due to the presence of PVP residue in the original membrane, N1s peak occurs at $399.8 \mathrm{eV}$, which is consistent with the result of the FTIR test. On the PSF-PDA/PEI membrane, the contents of $\mathrm{O}$ and $\mathrm{N}$ increased obviously. In addition, after reaction with epoxide, the $\mathrm{O}$ content increased on the membrane surface further. Table 1 shows elemental surface composition of original and modified membranes. We can see that the atomic ratio of $\mathrm{O} / \mathrm{N}$ in PSF-diol membrane is higher than that in the PSF-PDA/PEI membrane, which is consistent with the molecular structure of the membrane surface. This structure further proves that the composite membrane with a polyhydroxy structure on the surface was obtained by the co-deposition experiment. 


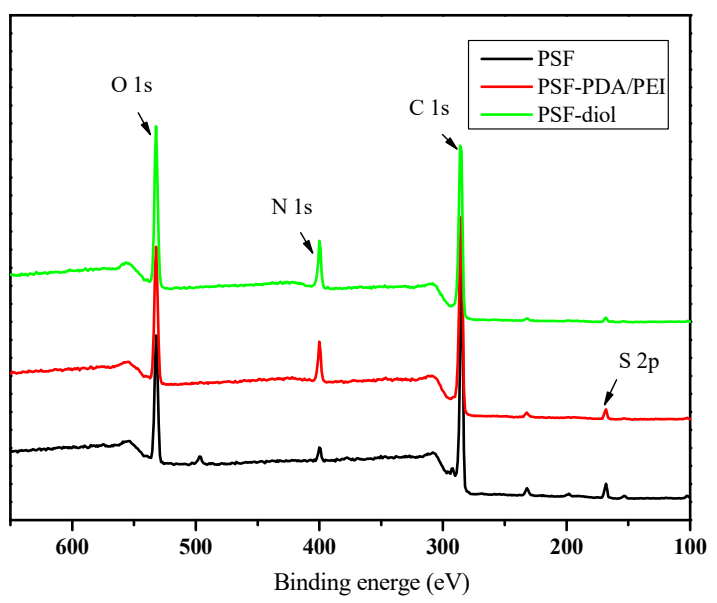

Figure 5. XPS spectra of PSF, PSF-PDA/PEI, and PSF-diol membranes.

Table 1. Elemental surface composition of original and modified membranes.

\begin{tabular}{cccccc}
\hline \multirow{2}{*}{ Membranes } & \multicolumn{3}{c}{ Atomic Percent (mol \%) } & Atomic Ratio \\
\cline { 2 - 6 } & $\mathbf{C}$ & $\mathbf{O}$ & $\mathbf{N}$ & $\mathbf{S}$ & O/N \\
\hline PSF & 72.61 & 18.45 & 3.51 & 2.7 & 5.26 \\
PSF-PDA/PEI & 70.97 & 19.55 & 7.83 & 1.64 & 2.50 \\
PSF-diol & 67.07 & 23.45 & 8.47 & 1 & 2.77 \\
\hline
\end{tabular}

\subsubsection{Surface and Cross-Sectional Morphologies of Membranes}

Figure 6 shows the surface and cross-sectional morphologies of PSF (Figure $6 a, a^{\prime}$ ), PSF-PDA/PEI (Figure $6 b, b^{\prime}$ ), and PSF-diol (Figure $6 c, c^{\prime}$ ) membranes. We can see their differences clearly from the outside. After the co-deposition of DA and PEI, the membrane pores on the surface of the composite membrane are smaller than those of the original film, and the outer surfaces of PSF-diol (Figure 6c) became denser than PSF (Figure 6a) and PSF-PDA/PEI (Figure 6b). With the introduction of DA and PEI on the membrane surface and the preparation of the composite membrane with a polyhydroxy structure, the hydrophilicity of the composite membrane was significantly improved, but at the same time, the presence of the coating reduced the membrane pores. In addition, no significant changes were observed from the membrane's section structure, indicating that the reaction mostly occurred on the membrane surface and had little effect on the section.

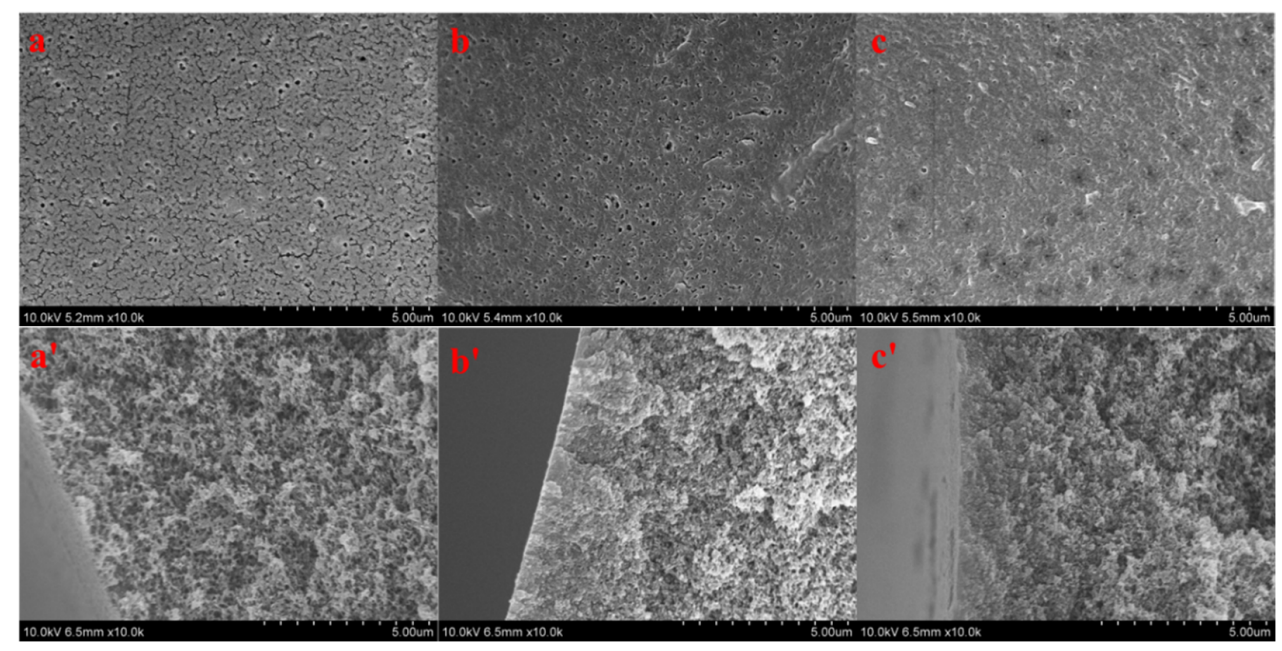

Figure 6. Surface and cross-sectional morphologies of PSF $\left(\mathbf{a}, \mathbf{a}^{\prime}\right)$, PSF-PDA/PEI $\left(\mathbf{b}, \mathbf{b}^{\prime}\right)$, and PSF-diol $\left(\mathbf{c}, \mathbf{c}^{\prime}\right)$ membranes. 


\subsubsection{Zeta Potential Tests}

Membrane zeta potential is used to characterize the amount of the charge density on the membrane surface [6]. Figure 7 shows the zeta potential of PSF-PDA/PEI and PSF-diol membranes within the $\mathrm{pH}$ range of 3-9. We can clearly observe that the PSF-PDA/PEI membrane has a significant positive charge, which is caused by the amino group on the surface. Shi et al. [28] pointed out that a PEI coating can obviously increase the positive charge of the membrane surfaces, which was due to the hydrolysis reaction of excess amine groups in PEI. After the reaction with glycidol, the potential PSF-diol is determined by the quaternary ammonium cations and hydroxide ion. The surface of the PSF-diol membrane is negatively charged due to the presence of a great quantity of hydroxyl groups [3].

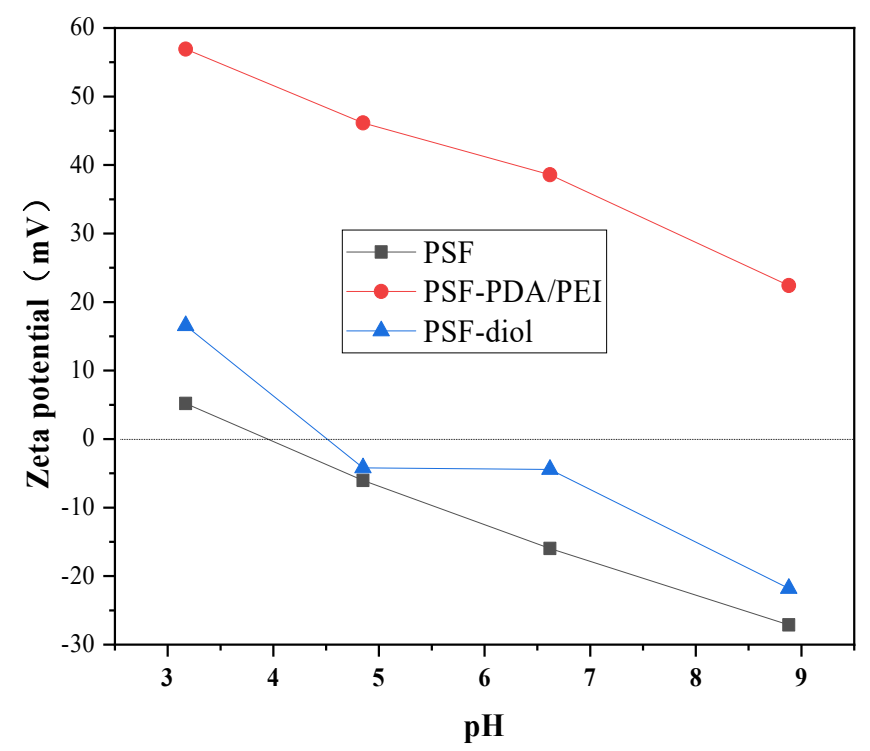

Figure 7. The zeta potential for PSF-PDA/PEI and PSF-diol membranes in different $\mathrm{pH}$.

\subsubsection{WCA Measurements}

In this paper, the static water contact angle test was used to determine the relative hydrophilicity of the membrane surface at least five locations. As seen in Figure 8, the WCA of origin membrane was $70^{\circ} \pm 5^{\circ}$, after coating by DA and PEI, the WCA of the PSF-PDA/PEI membrane reduced to about $45^{\circ} \pm 3^{\circ}$. The PSF-diol membrane had strong hydrophilicity and the WCA was an average of $38^{\circ}$. The results indicated that the hydrophilicity of the membrane surface was improved obviously after the introduction of hydrophilic functional groups such as amino and hydroxyl groups. The hydrophilicity of the membrane surface is related not only to the hydrophilic and hydrophobic properties of functional groups contained on the membrane surface but also to the roughness and electric charge of the membrane surface [29]. 


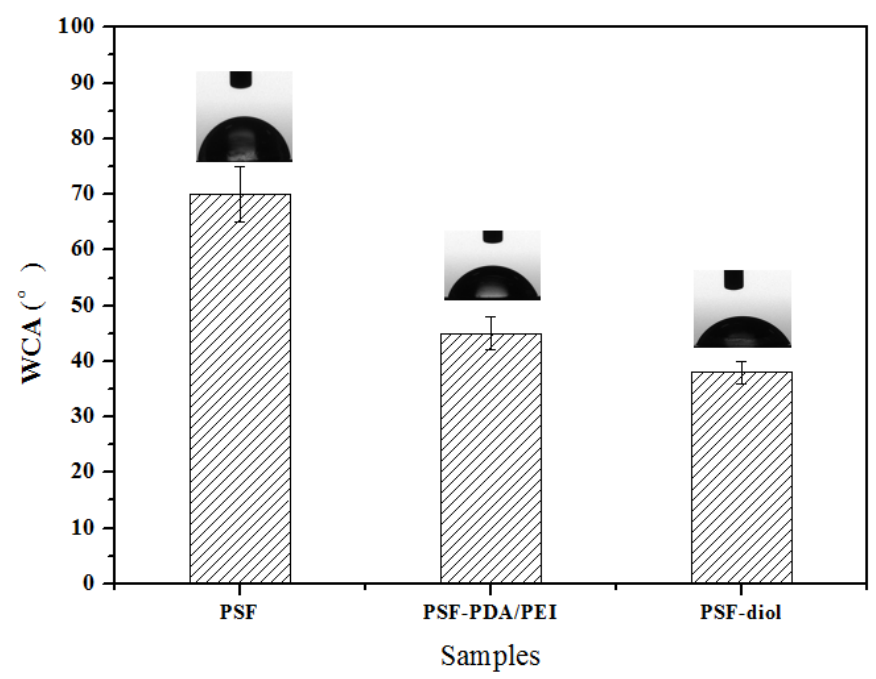

Figure 8. Water contact angle (WCA) of PSF, PSF-PDA/PEI, and PSF-diol membranes.

\subsection{Membrane Flux Performance}

The pure water fluxes of the original and modified membranes were measured at $0.1 \mathrm{MPa}, 25^{\circ} \mathrm{C}$. As can be seen from Figure 9, the original PSF membrane flux was $246 \mathrm{~L} / \mathrm{m}^{2} \mathrm{~h}$, while the water fluxes of PSF-PDA/PEI and PSF-diol membranes were 54 and $45 \mathrm{~L} / \mathrm{m}^{2} \mathrm{~h}$, respectively. Surface hydrophilicity is one of the main factors affecting water flux. The decrease of the water flux of the composite membrane was caused by the blockage of the membrane holes by the composite layer after the membrane surface was coated. Zin et al. [26] confirmed that the reaction between DA and PEI enhances the stability of the coating of membrane surface, which indicates that the coating of PDA/PEI on the surface of PSF membrane was successful.

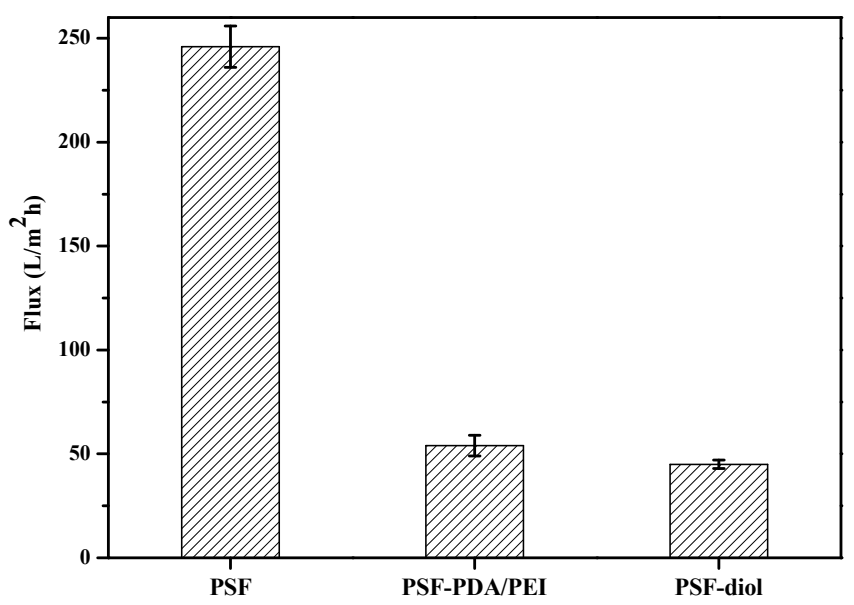

Figure 9. The flux of PSF, PSF-PDA/PEI, and PSF-diol membranes.

\subsection{Boron Removal Experiments}

The influence of initial boron concentration on the adsorption performance of PSF-diol membrane is shown in Figure 10. It can be seen from the figure that the adsorption amount of boric acid by PSF-diol membrane increases with the increase of initial boron concentration. When the initial concentration was $300 \mathrm{mg} / \mathrm{L}$, the maximum adsorption capacity appeared to be $1.61 \mathrm{mmol} / \mathrm{g}$, and then the adsorption platform appeared, this is because the surface of the PSF-diol membrane has a large number of ortho-hydroxyl groups that can be complexed with boric acid. 


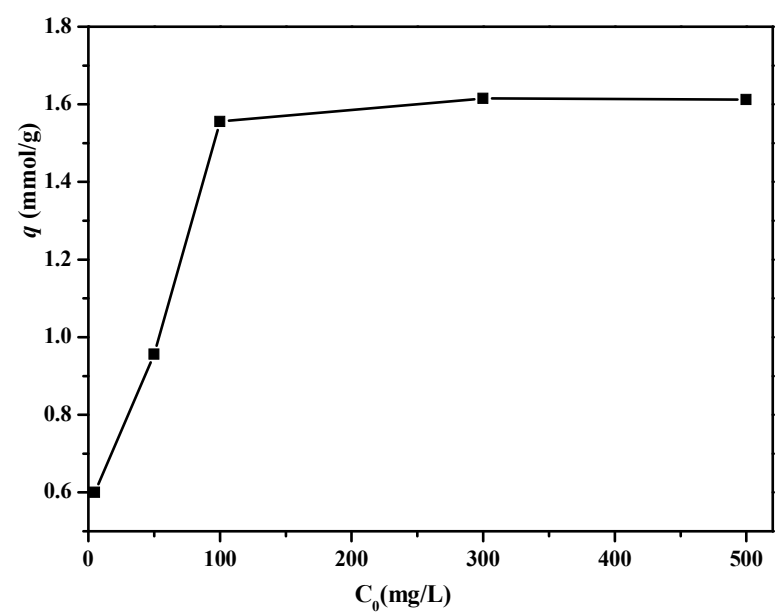

Figure 10. Effect of the initial boron concentration on boron uptake of PSF-diol membrane $(\mathrm{pH}=8$, $\left.\mathrm{T}=25^{\circ} \mathrm{C}\right)$.

In order to further understand the mechanism of boron adsorption, Langmuir and Freundlich isothermal adsorption models were used to fit the experimental data. The Langmuir isotherm model assumes a single-layer adsorption on a homogeneous surface. The Freundlich isothermal model assumes multilayer heterogeneous adsorption at different energy adsorption sites. Their equations are as follows:

$$
\begin{gathered}
\frac{C_{\mathrm{eq}}}{q}=\frac{C_{\mathrm{eq}}}{q_{\mathrm{max}}}+\frac{1}{b q_{\max }}, \\
q=K_{f} C_{\mathrm{eq}}^{1 / n},
\end{gathered}
$$

where, $q_{\max }(\mathrm{mmol} / \mathrm{g})$ and $C_{\text {eq }}(\mathrm{mg} / \mathrm{L})$ are the theoretical maximum adsorption capacity and adsorption concentration of the affinity membrane in the saturated state, respectively; $b(\mathrm{~L} / \mathrm{mg})$ is the adsorption coefficient, and $K_{f}$ and $1 / n$ are Freundlich constants (mmol/g).

Figure 11 shows the adsorption isotherm curves of the PSF-diol membrane, and the isotherm parameters are listed in Table 2. We can see from the results that compared with the Freundlich model, the $\mathrm{R}^{2}$ value fitted by Langmuir is the highest, and the calculated value of $q_{\max }$ is close to the experimental value, indicating that the adsorption of boric acid on the membrane surface is uniform monolayer adsorption.

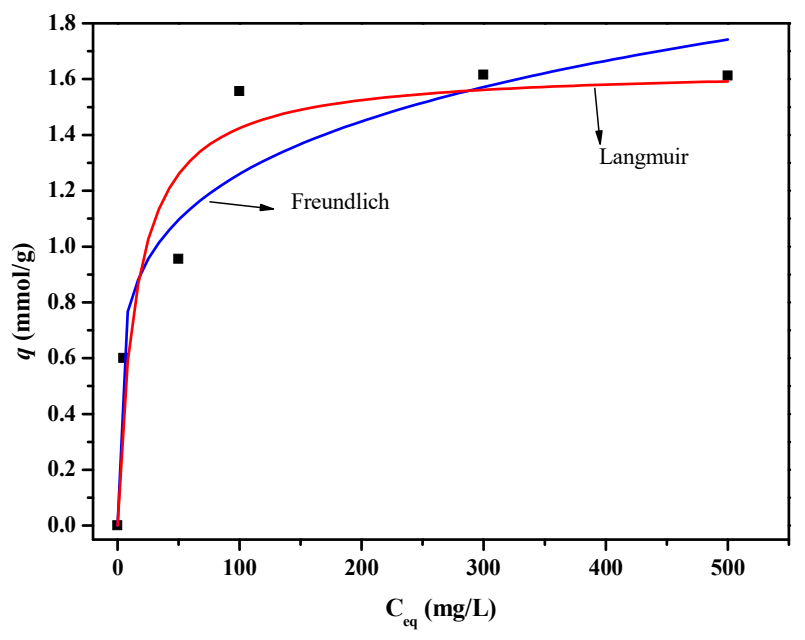

Figure 11. Boron adsorption isotherms of PSF-diol membrane. 
Table 2. List of isotherm parameters for boron adsorption $\left(\mathrm{pH}=8.0, \mathrm{~T}=25^{\circ} \mathrm{C}\right)$.

\begin{tabular}{ccccccc}
\hline \multirow{2}{*}{ Membrane } & \multicolumn{3}{c}{ Langmuir Constants } & \multicolumn{3}{c}{ Freundlich Constants } \\
\cline { 2 - 7 } & $\boldsymbol{q}_{\max }(\mathbf{m m o l} / \mathbf{g})$ & $\boldsymbol{b}(\mathbf{L} / \mathbf{m m o l})$ & $\mathbf{R}^{\mathbf{2}}$ & $\boldsymbol{K}_{f}(\mathbf{m m o l} / \mathbf{g})$ & $\mathbf{1} / \boldsymbol{n}$ & $\mathbf{R}^{\mathbf{2}}$ \\
\hline PSF-diol & 1.64 & 0.07 & 0.9322 & 0.49 & 0.20 & 0.8452 \\
\hline
\end{tabular}

As can be seen from Figure 12, the adsorption amount of boron increased sharply at the beginning, and then reached adsorption saturation at $10 \mathrm{~min}$. The results showed that the polyhydroxy functional groups on the affinity membrane surface can achieve a high efficiency of boron adsorption in a short time. As it is known, the adsorption kinetics of boric acid on the affinity membrane is mainly affected by boron diffusion between layers of the polyhydroxy copolymer on the surface of the affinity film and the interaction between boric acid and hydroxyl functional groups. Meng et al. [19] pointed out that the hyperbranched polymer scaffold is beneficial to superior boron-uptake performance.

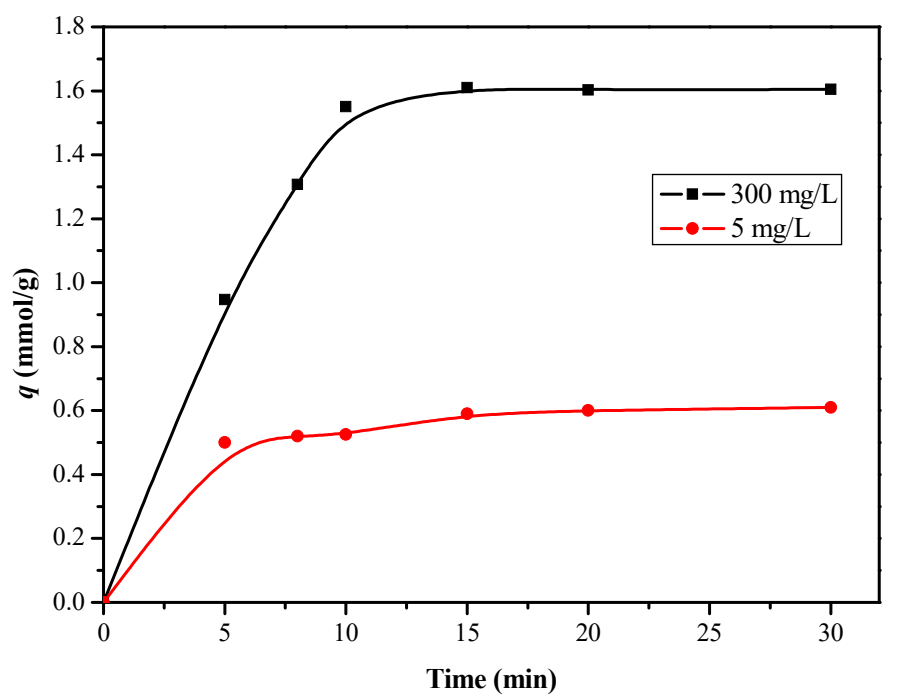

Figure 12. Effect of time on boron uptake of PSF-diol membrane $\left(\mathrm{pH}=8.0, \mathrm{~T}=25^{\circ} \mathrm{C}\right)$.

We investigated the effect of $\mathrm{pH}$ values on boron adsorption. It can be seen from Figure 13 that the composite membrane has a good adsorption effect on boric acid under weakly alkaline conditions. When the $\mathrm{pH}$ is 8.0 , the adsorption capacity reaches the maximum value. This is because under acidic conditions, there is a large amount of hydrogen ions in the solution, which will inevitably lead to its complexation reaction moving toward dissociation, and the adsorption capacity of boric acid decreases. Under alkaline conditions also, the adsorption capacity decreased because of the adsorption competition between borate ions and a large number of hydroxyl groups in the system.

Boron rejection percentage is an important parameter for evaluating adsorption performance in actual operation. Considering the actual content of boron in seawater, we chose the initial boron concentration of $5 \mathrm{mg} / \mathrm{L}$. The dynamic boron adsorption experiment of the modified membrane was studied by using a dead-end filter model. The device was connected to a peristaltic pump to control a constant flow rate of $0.5 \mathrm{~mL} / \mathrm{min}$, and the concentration of the permeable solution $\left(C_{\mathrm{p}}\right)$ was measured at certain volume intervals. The boron adsorption penetration curve is shown in Figure 14. It can be seen that the ratio of $C_{\mathrm{p}} / C_{0}$ changed rapidly at the beginning, when the volume of the filtrate reached $1 \mathrm{~mL}$, the ratio of $C_{\mathrm{p}} / C_{0}$ was 0.23 , which means the boron rejection percentage was $77 \%$. When the volume of the filtrate reached $6 \mathrm{~mL}$, the boron rejection performance did not fluctuate significantly, indicating a saturation adsorption, which is consistent with the results of the static boron adsorption experiment. 


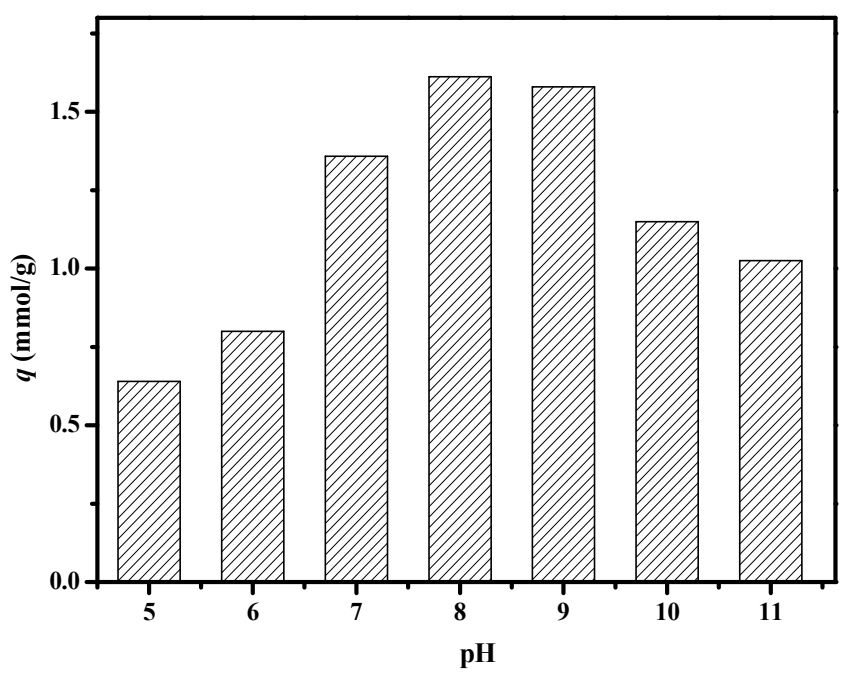

Figure 13. Effect of $\mathrm{pH}$ value on boron uptake of PSF-diol membrane $\left(C_{0}=300 \mathrm{mg} / \mathrm{L}, \mathrm{T}=25{ }^{\circ} \mathrm{C}\right)$.

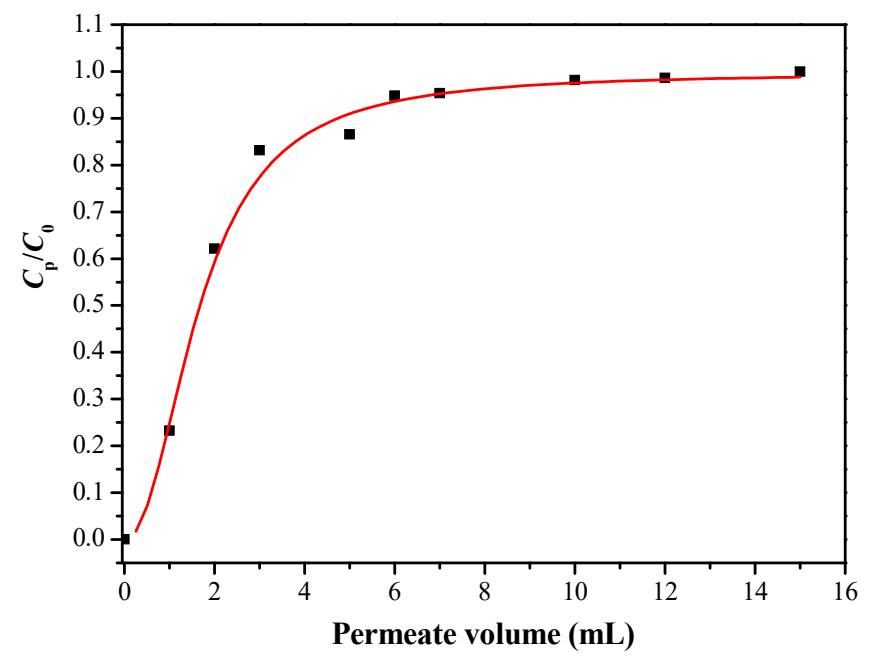

Figure 14. Breakthrough curve of PSF-diol membrane $\left(C_{0}=5 \mathrm{mg} / \mathrm{L}, \mathrm{pH}=8\right)$.

\section{Conclusions}

Through the co-deposition of DA and PEI on the surface of a PSF membrane, amine-rich surfaces were produced, then the DA/PEI-functionalized membranes were reacted with glycidol. The modified membranes of PSF-PDA/PEI and PSF-diol showed more hydrophilicity. Because the membrane surface was coated by a layer of polymer, the water flux of the prepared membrane decreased. The adsorption experiments on boric acid show that the PSF-diol membrane has high efficiency and fast adsorption of boron. The maximum adsorption capacity of boron appeared to be $1.61 \mathrm{mmol} / \mathrm{g}$ within $10 \mathrm{~min}$ at an initial boron content of $300 \mathrm{mg} / \mathrm{L}$. The Langmuir and Freundlich isothermal adsorption models were used to fit the experimental data. The results showed that the fitting value of the Langmuir model was closer to the experimental value, indicating that the adsorption process of boric acid on the membrane was homogeneous single-layer adsorption. Adsorption kinetics showed that saturation adsorption can be achieved in minutes at the initial content of $5 \mathrm{mg} / \mathrm{L}$, which is beneficial to a rapid filtration process. The composite membrane prepared in this paper has certain potential value in the field of boron removal in water treatment. However, the permeability of the composite membrane materials needs to be further improved. In the next step, we will improve the permeability of the composite membrane on the premise of ensuring the effect of boron removal. 
Author Contributions: Data curation, J.Y. (Jingkui Yang); investigation, K.Z.; methodology, S.Q. and M.H.; supervision, J.Y. (Jie Yu); writing—original draft, J.J.; writing—review and editing, X.D.

Funding: The authors gratefully acknowledge the Science and Technology Support Program of the Science and Technology Department of Guizhou province [2017]2808 and [2018]2183.

Conflicts of Interest: The authors declare no conflict of interest.

\section{References}

1. Wolska, J.; Bryjak, M. Methods for boron removal from aqueous solutions-A review. Desalination 2013, 310, 18-24. [CrossRef]

2. Rahmawati, K.; Ghaffour, N.; Aubry, C.; Amy, G.L. Boron removal efficiency from Red Sea water using different SWRO/BWRO membranes. J. Membr. Sci. 2012, 423, 522-529. [CrossRef]

3. Tang, Y.P.; Thong, L.; Chung, T.S. Recent advances in membrane materials and technologies for boron removal. J. Membr. Sci. 2017, 541, 434-446. [CrossRef]

4. Pieruz, G.; Grassia, P.; Dryfe, R.A.W. Boron removal from produced water by facilitated ion transfer. Desalination 2004, 167, 416-418. [CrossRef]

5. Sayiner, G.; Kandemirli, F.; Dimoglo, A. Evaluation of boron removal by electrocoagulation using iron and aluminum electrodes. Desalination 2008, 230, 205-212. [CrossRef]

6. Power, P.P.; Woods, G.W. The chemistry of boron and its speciation in plants. Plant Soil 1997, 193, 1-13. [CrossRef]

7. Wang, L.; Qi, T.; Gao, Z. Synthesis of N-methyl glucamine modified macroporous poly(GMA-co-TRIM) and its performance as a boron sorbent. React. Funct. Polym. 2007, 67, 202-209. [CrossRef]

8. Dydo, P.; Turek, M. Boron transport and removal using ion-exchange membranes: A critical review. Desalination 2013, 310, 2-8. [CrossRef]

9. Samatya, S.; Kabay, N.; Tuncel, A. Monodisper-porous N-methyl-D-glucamine functionalized poly(vinylbenzyl chloride-co-divinylbenzene) beads as boron selective sorbent. J. Appl. Polym. Sci. 2012, 126, 1475-1483. [CrossRef]

10. Jiang, B.; Zhang, X.; Zhao, X.; Li, F.Z. Removal of high level boron in aqueous solutions using continuous electrodeionization (CEDI). Sep. Purif. Technol. 2018, 192, 297-301. [CrossRef]

11. Nagasawa, H.; Iizuka, A.; Yamasaki, A. Utilization of bipolar membrane electrodialysis for the removal of boron from aqueous solution. Ind. Eng. Chem. Res. 2011, 50, 6325-6330. [CrossRef]

12. Kustin, K.; Pizer, R. Temperature-jump study of the rate and mechanism of the boric acid-tartaric acid complexation. J. Am. Chem. Soc. 1969, 91, 317-322. [CrossRef]

13. Yilmaz, A.E.; Boncukcuolu, R.; Bayar, S.; Fim, B.A.; Kocakerim, M. Boron removal by means of chemical precipitation with calcium hydroxide and calcium borate formation. Korean J. Chem. Eng. 2012, 29, 1382-1387. [CrossRef]

14. Wei, Y.; Zheng, Y.; Chen, J. Functionalization of regenerated cellulose membrane via surface initiated atom transfer radical polymerization for boron removal from aqueous solution. Langmuir 2011, 27, 6018-6025. [CrossRef]

15. Shi, Q.; Meng, J.; Xu, R.; Du, X.; Zhang, Y. Synthesis of hydrophilic polysulfone membranes having antifouling and boron adsorption properties via blending with an amphiphilic graft glycopolymer. J. Membr. Sci. 2013, 444, 50-59. [CrossRef]

16. Kamboh, M.A.; Yilmaz, M. Synthesis of N-methyl glucamine functionalized calyx [4] arene based magnetic poropollenin for the removal of boron from aqueous environment. Desalination 2013, 310, 67-74. [CrossRef]

17. Tural, S.; Ece, M.S.; Tural, B. Synthesis of novel magnetic nano-sorbent functionalized with $\mathrm{N}$-methyl-D-glucamine by click chemistry and removal of boron with magnetic separation method. Ecotoxicol. Environ. Saf. 2018, 162, 245-252. [CrossRef]

18. Du, X.; Meng, J.; Xu, R.; Shi, Q.; Zhang, Y. Polyol-grafted polysulfone membranes for boron removal: Effects of the ligand structure. J. Membr. Sci. 2015, 476, 205-215. [CrossRef]

19. Meng, J.; Cao, J.; Xu, R.; Wang, Z.; Sun, R. Hyperbranched grafting enabling simultaneous enhancement of the boric acid uptake and the adsorption rate of a complexing membrane. J. Mater. Chem. A 2016, 4, 11656-11665. [CrossRef] 
20. Wang, Z.; Wu, Z.; Zhang, Y.; Meng, J.Q. Hyperbranched-polyol-tethered poly (amic acid) electrospun nanofiber membrane with ultrahigh adsorption capacity for boron removal. Appl. Surface Sci. 2017, 402, 21-30. [CrossRef]

21. Yang, H.; Luo, J.; Lv, Y.; Shen, P.; Xu, Z. Surface engineering of polymer membranes via mussel-inspired chemistry. J. Membr. Sci. 2015, 483, 42-59. [CrossRef]

22. Luo, R.; Wang, X.; Deng, J.C. Dopamine-assisted deposition of poly (ethylene imine) for efficient heparinization. Colloids Surf. B Biointerfaces 2016, 144, 90-98. [CrossRef]

23. Ji, Y.; Qian, W.; An, Q.; Huang, S.; Lee, K.; Gao, C.J. Mussel-inspired zwitterionic dopamine nanoparticles as building blocks for constructing salt selective nanocomposite membranes. J. Membr. Sci. 2019, 572, 140-151. [CrossRef]

24. Wu, C.; Wang, H.; Wei, Z.; Li, C.; Luo, Z.D. Polydopamine-mediated surface functionalization of electrospun nanofibrous membranes: Preparation, characterization and their adsorption properties towards heavy metal ions. Appl. Surface Sci. 2015, 346, 207-215. [CrossRef]

25. Jiang, J.; Zhu, L.; Li, X.; Xu, Y.; Zhu, B.K. Surface modification of PE porous membranes based on the strong adhesion of polydopamine and covalent immobilization of heparin. J. Membr. Sci. 2010, 364, 194-202. [CrossRef]

26. Zin, G.; Wu, J.; Rezzadoria, K.; Petrusa, J.C.C.; Luccioa, M.D.; Li, Q.L. Modification of hydrophobic commercial PVDF microfiltration membranes into superhydrophilic membranes by the mussel-inspired method with dopamine and polyethyleneimine. Sep. Purif. Technol. 2019, 212, 641-649. [CrossRef]

27. Jin, J.; Zhang, K.; Du, X.; Yang, J. Synthesis of polydopamine-mediated PP hollow fibrous membranes with good hydrophilicity and antifouling properties. J. Appl. Polym. Sci. 2017, 134, 44430. [CrossRef]

28. Shi, H.; Xue, L.; Gao, A.; Fu, Y.; Zhou, Q.; Zhu, L. Fouling-resistant and adhesion-resistant surface modification of dual layer PVDF hollow fiber membrane by dopamine and quaternary polyethyleneimine. J. Membr. Sci. 2016, 498, 39-47. [CrossRef]

29. Jin, J.; Liu, D.; Zhang, D.; Yin, Y.; Zhao, X.; Zhang, Y. Preparation of thin-film composite nanofiltration membranes with improved antifouling property and flux using 2,2'-oxybis-ethylamine. Desalination 2015, 355, 141-146. [CrossRef] 